\title{
Rapid detection of benzimidazole resistance in Botrytis cinerea by loop- mediated isothermal amplification
}

\author{
Fei Fan ${ }^{1}$, Matthias Hahn², Guo-Qing Li ${ }^{3}$, Yang Lin ${ }^{1,3}$ and Chao-Xi Luo ${ }^{1,3^{*}}$ (D)
}

\begin{abstract}
Benzimidazole fungicides (MBCs) have been widely used in agriculture since 1970s, and resistance to this class of fungicides in Botrytis cinerea is reported worldwide. Resistance to MBCs in B. cinerea is related to mutations in the target $\beta$-tubulin gene (TUB2). Compared with the mutation at codon 200, the substitutions from glutamic acid to alanine (E198A), valine (E198V), or lysine (E198K) at codon 198 are currently the predominant mutations in MBC resistant populations of $B$. cinerea. In this study, a loop-mediated isothermal amplification (LAMP) method was established for rapid detection of benzimidazole resistance in $B$. cinerea. On the basis of the three mutations at TUB2 codon 198, three sets of LAMP primers were designed, and each of these primer sets was able to specifically amplify the DNA containing its corresponding mutation, while no amplification was detected with other mutated or the wild type DNA. After optimization, the sensitivity and specificity tests illustrated that this LAMP assay had good sensitivity and specificity to detect specific resistant genotypes in B. cinerea. Result also showed that the LAMP assay had good reproducibility. Above all, boiled mycelia could be used as templates, which simplified the process and increased the efficiency of the assay. Considering its rapidity, simplicity, and high efficiency, the LAMP assay developed in this study is a promising tool for the diagnosis of benzimidazole resistance in B. cinerea, and will contribute to the monitoring of resistance development to MBCs in the future.
\end{abstract}

Keywords: Botrytis cinerea, Fungicide resistance, Benzimidazole fungicide (MBC), Loop-mediated isothermal amplification (LAMP), Mutation of TUB2

\section{Background}

Grey mold is a common disease on numerous crops worldwide, especially on vegetables and small fruits. The causal agent of this disease is the ascomycete fungus Botrytis cinerea (Williamson et al. 2007). To control this fungus, fungicides are widely used. At present, several classes of fungicides are available, and the group of benzimidazole fungicides (MBCs) is one of them. MBCs, including carbendazim, benomyl and the related agent thiophanate-methyl, are broad-spectrum fungicides and the first group of systemic single-site fungicide

\footnotetext{
*Correspondence: cxluo@mail.hzau.edu.cn

'Key Lab of Horticultural Plant Biology, Ministry of Education, and College of Plant Science and Technology, Huazhong Agricultural University, Wuhan 430070, China

${ }^{3}$ College of Plant Science and Technology and the Key Lab of Crop Disease Monitoring \& Safety Control in Hubei Province, Huazhong Agricultural University, Wuhan 430070, China

Full list of author information is available at the end of the article
}

introduced into agriculture in the early 1970s (Walker et al. 2013). MBCs bind to $\beta$-tubulin and prevent microtubule assembly, thereby inhibiting germ-tube elongation and mycelial growth (Leroux et al. 1999).

Soon after the introduction, resistance to this class of fungicides in B. cinerea was reported (Bolton 1976; Tripathi and Schlösser 1982). This resistance was related to several mutations in the $\beta$-tubulin gene (TUB2) at codon 198, which resulted in amino acid replacement of glutamic acid by alanine (E198A), valine (E198V), or lysine (E198K) (Yarden and Katan 1993; Banno et al. 2008). Moreover, a mutation at codon 200 substituting phenylalanine by tyrosine (F200Y) was also involved in the resistance to $\mathrm{MBC}$ fungicides (Yarden and Katan 1993). Interestingly, apart from benzimidazole resistance, mutations E198K and F200Y also conferred resistance to another anti-microtubule fungicide, diethofencarb, while strains with E198A and E198V mutations were sensitive

(c) The Author(s). 2019 Open Access This article is distributed under the terms of the Creative Commons Attribution 4.0 International License (http://creativecommons.org/licenses/by/4.0/), which permits unrestricted use, distribution, and 
to diethofencarb (Yarden and Katan 1993; Banno et al. 2008). In Japanese fields, most of benzimidazole-resistant B. cinerea isolates had the E198V or E198A mutation (Bardas et al. 2008). Our earlier research also showed that mutations at codon 198 were predominant in the isolates with resistance to carbendazim in Hubei, China (Fan et al. 2016; Fan et al. 2017).

To manage fungicide resistance, information on the occurrence and prevalence of resistance to registered fungicides is particularly significant for crop producers to adjust their management strategy promptly. Current methods for detection of MBC resistance in B. cinerea include the determination of $\mathrm{EC}_{50}$ values, visual analysis of growth on medium amended with a single discriminatory concentration of an MBC fungicide, and allele-specific polymerase chain reaction (Luck and Gillings 1995; Fernández-Ortuño et al. 2014). Additionally, real-time PCR, PCR-restriction-fragment length polymorphism (PCR-RFLP), and the two-step high-resolution melting (HRM) analysis were also applied in benzimidazole resistance identification (Banno et al. 2008; Ziogas et al. 2009; Chatzidimopoulos et al. 2014). Detection of resistance based on $\mathrm{EC}_{50}$ determination or discriminatory dose consumes time and labor, and cannot identify the point mutations, while modern molecular techniques mentioned require DNA extraction, thermal cycling instruments, and other sophisticated equipment. Therefore, these methods are not suitable for on-site detection.

A technique that has the potential to provide rapid, yet more feasible in-field detection of pathogens is loop-mediated isothermal amplification (LAMP). As a novel method of DNA amplification, LAMP combines high specificity, efficiency and rapidity (Notomi et al. 2000). Compared with Polymerase Chain Reaction (PCR), LAMP obviates the demand for a thermal cycler, due to isothermal conditions. Furthermore, the LAMP product can be visualized by several methods, such as staining with SYBR Green I, ethidium bromide (EB), hydroxynaphthol blue (HNB) and calcein or using generic lateral flow devices (Tomita et al. 2008; Goto et al. 2009; Tomlinson et al. 2010b). Observing turbidity increase derived from magnesium pyrophosphate formation is also a choice to analyze LAMP product (Njiru 2012). Because of the advantages of LAMP, it has been widely applied in field studies, especially for the diagnosis of bacteria, viruses, fungi, and transgenic plants (Niessen and Vogel 2010; Wang et al. 2012; Zhou et al. 2014; Kuan et al. 2016). Recently, LAMP has been applied successfully to detect fungicide and herbicide resistance, such as carbendazim resistance in Fusarium graminearum and Sclerotinia sclerotiorum (Duan et al. 2014a; Duan et al. 2015), and fenoxaprop-p-ethyl resistance in American slough grass (Pan et al. 2015). Even though this remarkable technique has been applied for the detection of $B$. cinerea (Duan et al. 2014b), it has not been adopted to detect MBC resistance in $B$. cinerea.

Here, we established a LAMP assay for rapid detection of benzimidazole resistant isolates of $B$. cinerea based on point mutations at codon 198 of TUB2. Based on three mutations (E198A, E198V, and E198K), three sets of LAMP primers were designed. Our study showed that each of these three sets of primers was able to specifically amplify the DNA containing its corresponding mutation, while no amplification was detected with other mutations or the wild type DNA. It was demonstrated that the established LAMP assay had good sensitivity, specificity and reproducibility. Furthermore, the LAMP assay could be accomplished using boiled mycelia as templates, which made this assay simple and time-saving. We believe that this LAMP assay is a promising tool for the detection of benzimidazole resistance in $B$. cinerea, and will play a significant role in monitoring resistance against MBCs in the future.

\section{Results \\ Design of LAMP primers}

Using the sequence of TUB2 with the E198V point mutation, eight sets of LAMP primers were designed. Only one set of LAMP primers named Tub-E198V (Fig. 1a and Table 1) was able to identify the E198V mutation. To increase the primer specificity, the position of $\mathrm{F} 2$ primer was changed to end with the mutation, and one nucleotide mismatch was introduced before the mutation (Fig. 1a and Table 1). After addition of SYBR Green I, the samples of E198A, E198K, sensitive isolate, and DNA-free control remained brown, but the sample of E198V changed to a fluorescent yellow color (Fig. 2a), which indicated the positive LAMP reaction. Agarose gel electrophoresis showed that this set of primers could only amplify DNA from the E198V isolate, but not from any other mutated isolates or the sensitive isolates (Fig. 2b). After the successful design of Tub-E198V, the F2 primer was modified to fit the other two mutations (E198A and E198K) following the same strategy (Fig. 1 and Table 1). Both dye treatment and electrophoresis demonstrated that primer sets Tub-E198A and Tub-E198K were capable of specifically recognizing their corresponding mutations respectively (Fig. 2).

\section{Optimization of LAMP reaction}

The LAMP reaction system was optimized using the primer set Tub-E198V and the corresponding genomic DNA. The optimized LAMP master mix contained $4 \mathrm{U}$ of Bst DNA polymerase, $2.5 \mu \mathrm{L} 10 \times$ ThermoPol Buffer, $4 \mathrm{mM} \mathrm{MgSO}_{4}, 1 \mathrm{mM}$ dNTPs, $1.2 \mathrm{mM}$ each of primers FIP and BIP, $0.4 \mathrm{mM}$ each of primers $\mathrm{F} 3$ and B3, $0.8 \mathrm{M}$ betaine, and $1 \mu \mathrm{L}$ of target DNA in a final volume of $25 \mu \mathrm{L}$. Best results were obtained when LAMP reaction was performed at $63^{\circ} \mathrm{C}$ for $60 \mathrm{~min}$. 


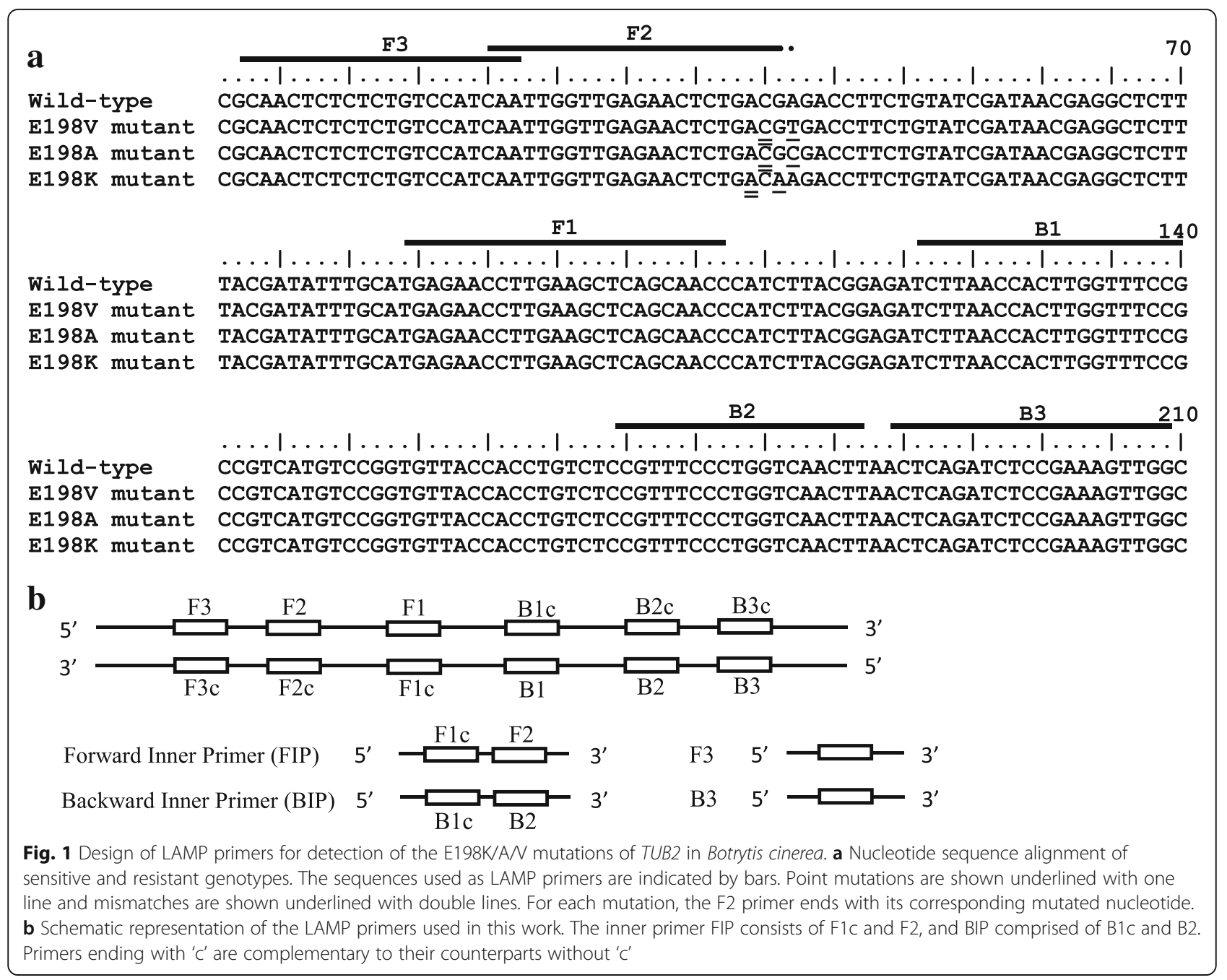

Sensitivity of LAMP and conventional PCR

The sensitivity of LAMP and conventional PCR was investigated using Tub-E198V primer set and ten-fold serial dilutions of plasmid pMD18-TubA. When the concentration of plasmid reached $2 \times 10^{5}$ copies $/ \mu \mathrm{L}$, the ladder-like product was observed by electrophoresis, and the color change of SYBR Green I also occurred
(Fig. 3a, b), indicating that the LAMP detection limit for plasmid pMD18-TubA was approximately $2 \times 10^{5}$ copies $/ \mu \mathrm{L}$. For conventional PCR, a $936 \mathrm{bp}$ band was amplified when the template plasmid was diluted to as low as $2 \times 10^{3}$ copies/ $\mu$ L (Fig. 3c), illustrating that the detection limit of PCR was about two orders of magnitude lower than that of LAMP.

Table 1 Design of LAMP primers for detection of the E198KAN mutations of TUB2 in Botrytis cinerea

\begin{tabular}{|c|c|c|c|}
\hline Primer & Type & Length (bp) & Sequence $\left(5^{\prime}-3^{\prime}\right)$ \\
\hline F3 & Forward outer & 21 & GCAACTCTCTCTGTCCATCAA \\
\hline B3 & Backward outer & 21 & CCAACTITCGGAGATCTGAGT \\
\hline BIP & Backward inner(B1c-B2) & 42 & TCTTAACCACTTGGTTTCCGCCGAAGTTGACCAGGGAAACGG \\
\hline Tub-E198V & Forward inner(F1c-F2) & 47 & GGTTGCTGAGCTTCAAGGTTCTCACAATTGGTTGAGAACTCTGAG ${ }^{\mathrm{b}} \mathrm{GT}^{\mathrm{2}}$ \\
\hline Tub-E198A & Forward inner(F1C-F2) & 47 & GGTTGCTGAGCTTCAAGGTTCTCACAATTGGTTGAGAACTCTGAT ${ }^{\mathrm{b}} \mathrm{GC}$ \\
\hline Tub-E198K & Forward inner(F1C-F2) & 46 & GGTTGCTGAGCTTCAAGGTTCTCACAATTGGTTGAGAACTCTGI ${ }^{\mathrm{b}} \mathrm{CA}^{\mathrm{a}}$ \\
\hline
\end{tabular}

${ }^{a}$ Necleotides in bold are modified from the sequence of the TUB2 gene in the sensitive and the resistant isolates

${ }^{b}$ Necleotides underlined are mismatches introduced specifically to distinguish B.cinerea genotypes (E198K/A/V) 


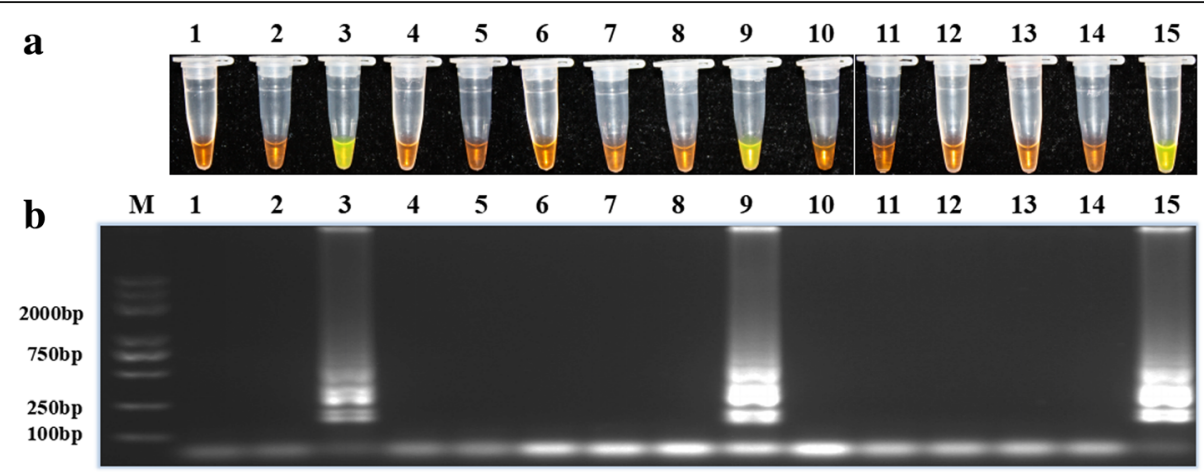

Fig. 2 LAMP primer set designed to amplify the E198K, E198A and E198V mutations in Botrytis cinerea, respectively. a LAMP products detected with $1000 \times$ SYBR Green I. b Detection of LAMP products by agarose gel electrophoresis. Lane M: DNA $2 \mathrm{~K}$ plus Marker; tubes and lanes 1-5: primer set Tub-E198K; tubes and lanes 6-10: primer set Tub-E198A; tubes and lanes 11-15: primer set Tub-E198V; tubes and lanes 1, 6, 11: ddH ${ }_{2} \mathrm{O}$; tubes and lanes 2, 7, 12: HBStr-104; tubes and lanes 3, 8, 13: HBStr-155; tubes and lanes 4, 9, 14: HBStr-114; tubes and lanes 5, 10, 15: HBStr-496

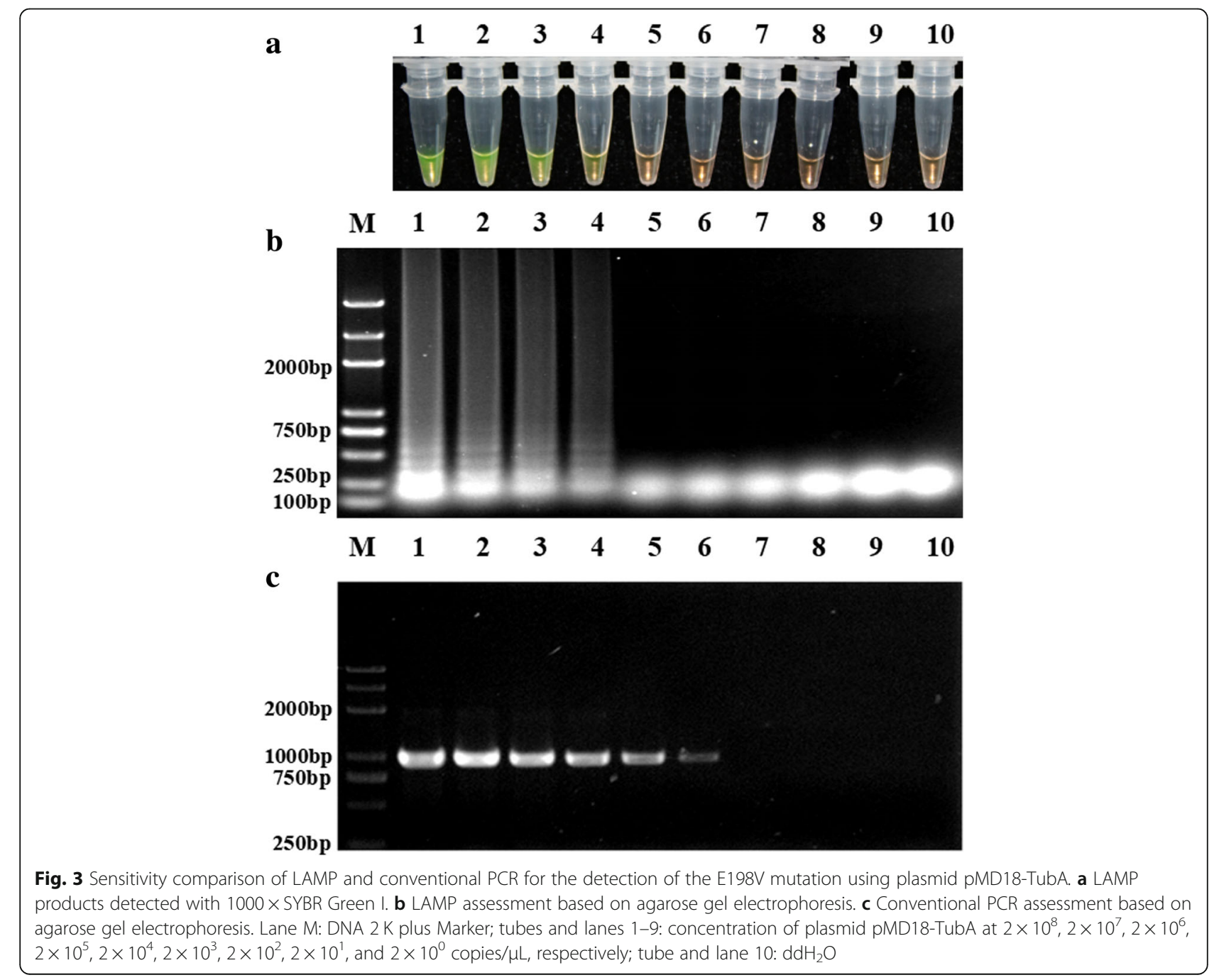




\section{Specificity and reproducibility test of LAMP}

Genomic DNA was extracted from several relative species of $B$. cinerea, including $B$. sinoviticola, B. sinoallii, B. porri, S. sclerotiorum, Monilinia fructicola, M. mumecola, M. yunnanensis, and F. graminearum, and the specificity of LAMP by using Tub-E198V primer set was subsequently tested. Both the color change in the tubes (Fig. 4a) and amplification bands (Fig. 4b) were only achieved with DNA of carbendazim-resistant $B$. cinerea isolates with E198V mutation, confirming the high specificity of LAMP.

To evaluate the reproducibility, all three LAMP primer sets were employed against genomic DNA from eight isolates for each of wild type, E198V, E198A and E198K mutant, respectively. Using primer set Tub-E198V, only the eight E198V resistant isolates showed positive reaction, whereas the reaction of the eight sensitive isolates, eight E198A resistant isolates, and eight E198K resistant isolates remained negative (Additional file 1: Figure S1). As expected, similar observations were made using primer sets Tub-E198A and Tub-E198K (Additional file 2: Figure S2 and Additional file 3: Figure S3).

\section{LAMP detection using crude DNA from fungal mycelia} Outside the laboratory, DNA extraction may be a limiting step for the LAMP assay. To solve this problem and to increase the speed and economic efficiency, crude DNA extracted with $10 \times$ TE buffer from mycelia was used as the template in LAMP detection. After LAMP amplification with primer set Tub-E198V, the fluorescent yellow of SYBR Green I occurred only with mycelia of four E198V resistant isolates, whereas SYBR Green I stayed brown for sensitive isolates and the resistant isolates with the E198A or E198K mutation (Fig. 5a), which was also validated by agarose gel electrophoresis (Fig. 5b). The results showed that the LAMP detection could also be successful when crude mycelial extracts were used as template to detect the carbendazim resistance conferred by E198A/V/K mutations in B. cinerea.

\section{Discussion}

MBCs, such as carbendazim and thiophanate-methyl, have been used in agriculture since 1970s (Walker et al. 2013). They inhibit germ-tube elongation and mycelial growth by targeting $\beta$-tubulin, preventing microtubule assembly (Leroux et al. 1999). Due to the specific-site mode of action, the fungus can develop resistance based on single mutations. By now, four mutations in TUB2 gene are known to be involved in resistance to $\mathrm{MBCs}$ in B. cinerea, namely E198A, E198V, E198K, and F200Y (Yarden and Katan 1993; Banno et al. 2008). Compared with the extremely rare occurrence of mutation at codon 200, mutations at codon 198 were found at a high frequency (Banno et al. 2008). In our previous studies, all resistant mutations occurred at codon 198 in sequenced isolates from Hubei Province, China (Fan et al. 2016; Fan et al. 2017). Interestingly, although four types of mutations conferred resistance to benzimidazoles, only mutations E198K and F200Y are capable of conferring resistance to both MBC fungicide and diethofencarb (Yarden and Katan 1993; Banno et al. 2008). Diethofencarb, belonging to $N$-phenylcarbamate, is another anti-microtubule fungicide but negative cross resistance was observed between diethofencarb and $\mathrm{MBC}$

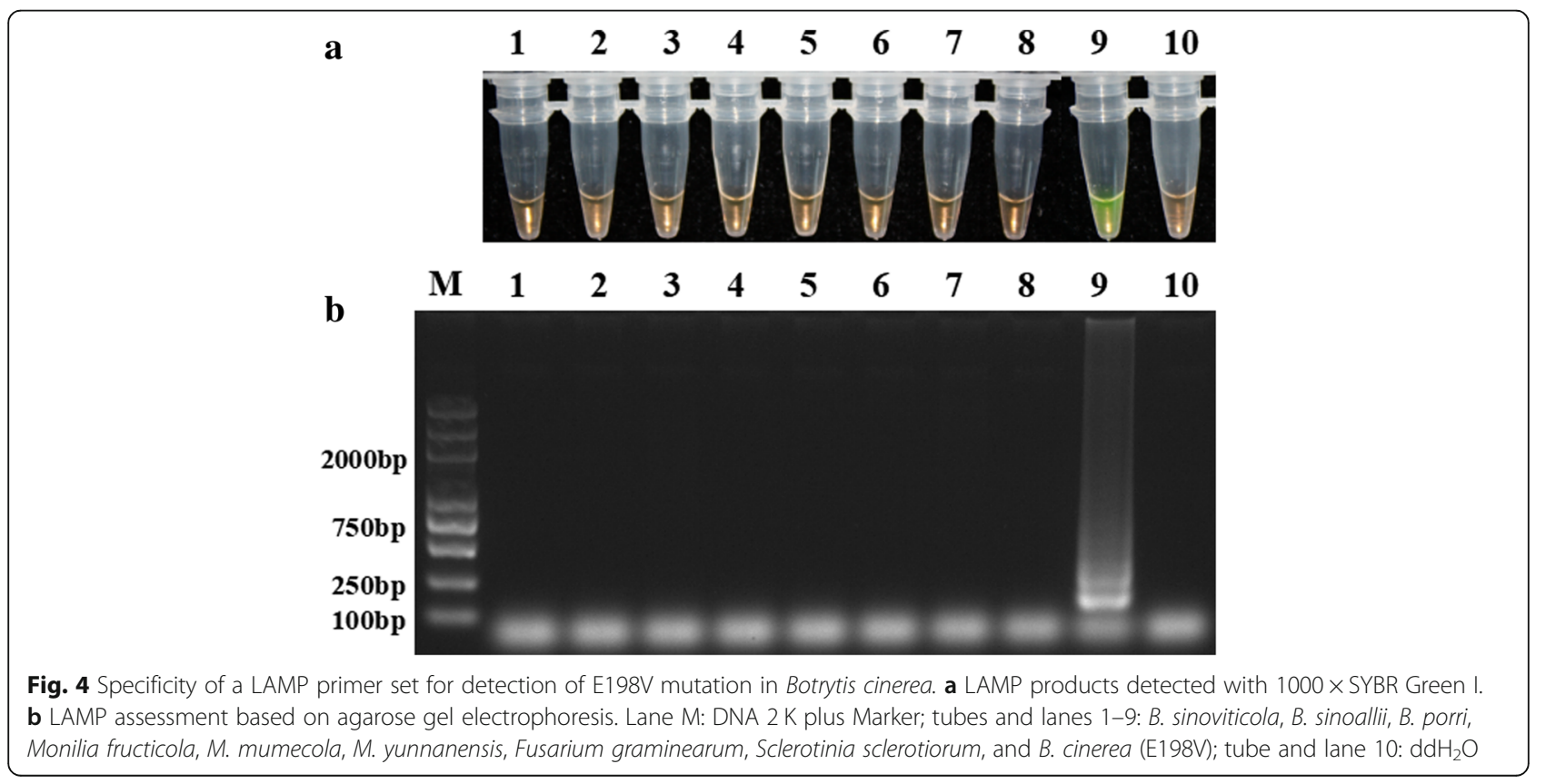


$\mathbf{a}$
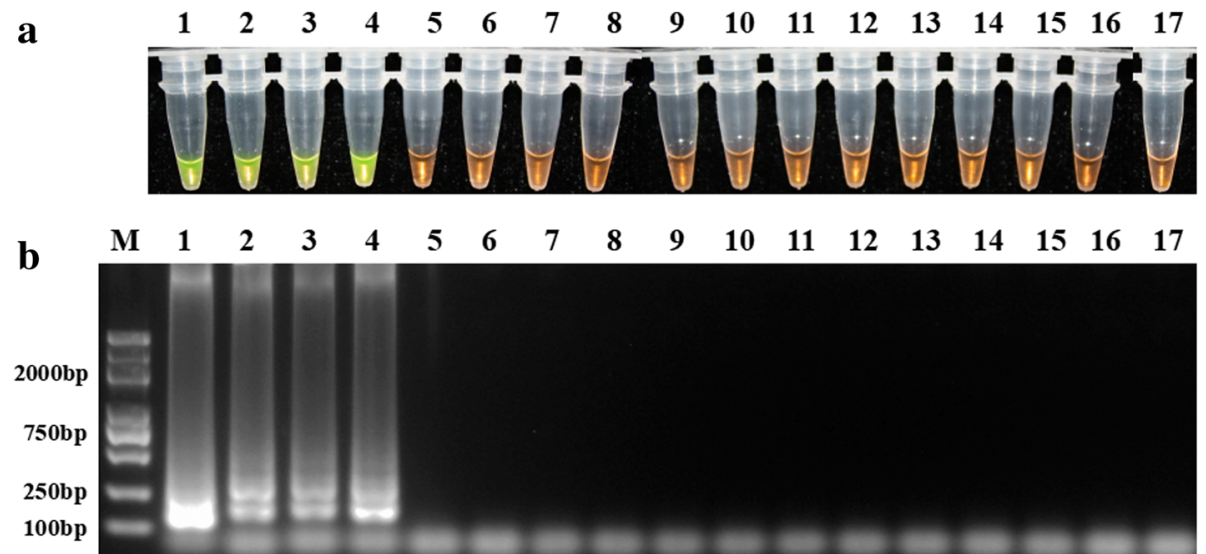

Fig. 5 Conduction of LAMP using mycelia. a LAMP products detected with $1000 \times$ SYBR Green I. $\mathbf{b}$ Detection of LAMP products by agarose gel electrophoresis. Lane M: DNA 2 K plus Marker; tubes and lanes 1-4: E198V strains; tubes and lanes 5-8: E198K strains; tubes and lanes 9-12: E198A strains; tubes and lanes 13-16: wild type strains; tube and lane 17: $\mathrm{ddH}_{2} \mathrm{O}$

fungicides in isolates with E198A or E198V mutation (Leroux et al. 1999).

Resistance monitoring, an indispensable step of resistance management, is valuable to guide the efficient use of fungicides. For resistance detection, growth assays on fungicide-amended media can be used, which consume time and labor, and do not allow identification of the resistance mutations. Modern molecular tools depending on PCR, such as conventional PCR, allele-specific PCR, real-time PCR, and PCR-RFLP, show some advantages over classic methods, but sophisticated equipment, reagents and skilled personnel are required. The technique based on loop-mediated isothermal amplification (LAMP) established in this paper for identification of $\mathrm{MBCs}$ resistance is specific, rapid, simple and efficient, and has the potential to be conducted in the field, which facilitates the efficient use of fungicides.

According to a previous study, LAMP is highly specific due to its six distinct primers (Notomi et al. 2000). In this study, LAMP primers designed using the TUB2 gene sequence of $B$. cinerea could specifically amplify the DNA from resistant strains of $B$. cinerea. Such a high specificity of LAMP was also documented in other studies (Duan et al. 2014a; Duan et al. 2015). Moreover, LAMP was also found to be highly sensitive compared with conventional PCR (Notomi et al. 2000; Zhou et al. 2014; Pan et al. 2015). However, our experiments revealed that the detection limit of LAMP was approximately 100 times lower than that of conventional PCR. It is possible that the sensitivity of LAMP is influenced by the affinity of specific primers and target DNAs. Even though the LAMP assay developed in this study was not as sensitive as that in other studies, the sensitivity was still high enough for detection, even using the crude DNA obtained from boiled mycelia. This is an important simplification of LAMP detection, since this method does not require sophisticated equipment or expensive reagent. Considering that there are large amounts of mycelia on diseased samples of grey mold, no isolation and cultivation of $B$. cinerea is required for the LAMP assessment.

The LAMP reaction can be accelerated by the addition of two loop primers, named loop forward (LF) primer and loop backward (LB) primer (Nagamine et al. 2002). By now, loop primers have been successfully applied in many studies (Tomlinson et al. 2010a; Wang et al. 2011; Moradi et al. 2012). However, with the loop primers, the LAMP assay failed to distinguish the resistant mutants from the wild type of $F$. graminearum in a previous study (Duan et al. 2014a). Similarly, LAMP assay with the loop primer could not differentiate the resistant mutation from wild type when the reaction time was increased to $45 \mathrm{~min}$ (Duan et al. 2016). It seems that the LAMP analysis with loop primers can lead to false positive reaction when conducting resistance monitoring. According to the manual for LAMP primer designing, the loop primers are not essential. Therefore, similar to other studies (Pan et al. 2015; Duan et al. 2016), the loop primers were not adopted in this study.

As LAMP is a promising method for diagnosis of pathogens, it continues to attract the attention of researchers in different fields (Mori et al. 2013). Still, LAMP technique has a major problem, the false positive results arising from product cross contamination (Njiru et al. 2008; Angamuthu et al. 2012; Li et al. 2014). Contamination was usually caused by aerosol of product, especially when analyzing the LAMP product (Zhou et al. 2014). To avoid contamination, various methods have been developed (Goto et al. 2009; Zoheir and Allam 2011; Karthik et al. 2014). In our study, LAMP reaction mixture was prepared in a laminar flow cabinet, and the detection area was separated from preparation and 
amplification areas. In this way, false positive amplification was largely avoided. Since all the three sets of LAMP primers shared the same primer set, we were wondering if they could work together in a single reaction to facilitate the detection of MBCs resistance. Although multiplex PCR has been successfully applied in many studies (Côté et al. 2004; Hu et al. 2011), there has been no study on multiplex LAMP. In the current study, a lot of efforts were expended. Unfortunately, when using all the primers simultaneously, no DNA amplification was detected (data not shown). The complex structures formed among the primers might inhibit the reaction. Further attempts will be made to achieve the goal of multiplex LAMP.

The development of fungicide resistance in $B$. cinerea populations is one of the major limiting factors of successful control of gray mold (Hahn 2014). Resistance to MBCs in B. cinerea is reported worldwide (Zhang et al. 2010; Weber 2011; Fernández-Ortuño et al. 2014). In order to provide guidance on how to use MBCs efficiently, continuous monitoring of fungicide resistance is necessary. Since the mutations at codon 198 are predominant in benzimidazole-resistant isolates, the LAMP method established in the present study could be easily adopted as a simple, rapid and efficient method to identify resistant isolates of $B$. cinerea, especially by using templates directly from mycelia.

\section{Conclusions}

Resistance to MBCs in B. cinerea is related to the mutations in the $\beta$-tubulin gene, and the mutations E198A/ $\mathrm{V} / \mathrm{K}$ are predominant genotypes. For rapid detection of benzimidazole resistance caused by the E198A/V/K mutations, a LAMP method was established in this study. Results showed that this LAMP method had good sensitivity and specificity, as well as good reproducibility. Above all, boiled mycelia could be used as LAMP templates. Considering its rapidity, simplicity, and high efficiency, this LAMP assay is a promising tool for the detection of MBCs resistance in B. cinerea, and will significantly contribute to the monitoring of resistance development to $\mathrm{MBCs}$ in practice.

\section{Methods}

\section{Fungal isolates and DNA extraction}

Four isolates of B. cinerea, HBstr-104, HBstr-114, HBstr-155, and HBstr-496 were tested in this study. They were collected from greenhouse strawberries in 2012 in Hubei Province, China. DNA sequencing showed that HBstr-114, HBstr-155, and HBstr-496 contained the point mutation of E198A (GAG to GCG), E198K (GAG to AAG), and E198V (GAG to GTG) in TUB2, respectively. As a consequence, isolates HBstr-114, HBstr-155, and HBstr-496 were resistant to benzimidazole fungicide carbendazim. Isolate HBstr-104 was used as a sensitive control. In the specificity test for the LAMP primers, several relative species of $B$. cinerea were tested, including B. sinoviticola, B. sinoallii, $B$. porri, S. sclerotiorum, M. fructicola, M. mumecola, $M$. yunnanensis, and $F$. graminearum. In reproducibility test, $32 \mathrm{~B}$. cinerea isolates including eight for each of E198V, E198K, E198A and wild type strains were tested. All isolates were cultured on potato dextrose agar (PDA) medium at $22^{\circ} \mathrm{C}$ in darkness.

To extract the genomic DNA, isolates were grown on PDA with cellophane for 3 days, and mycelia were collected using a toothpick. The genomic DNA was extracted and purified as described previously (Chi et al. 2009). The final concentration of extracted DNA was around $100 \mathrm{ng} / \mu \mathrm{L}$.

\section{Primer design}

On the basis of the DNA sequence of the E198V mutation, a set of LAMP primers named Tub-E198V (Table 1) was designed using the Primer Explorer V4 software program (http://primerexplorer.jp/elamp4.0.0/index.html). Depending on this primer set, two new LAMP primer sets Tub-E198A and Tub-E198K were also designed to distinguish the other two mutations. The three primer sets shared all the same primers except primer F2, which was modified based on each mutation. Furthermore, a mismatch was introduced in primer F2 to avoid false positives.

\section{Reaction mixture for LAMP}

The initial LAMP reaction was performed according to a former study, with a little adjustment (Duan et al. 2014a). The LAMP reaction was carried out in a $25 \mu \mathrm{L}$ volume containing $6 \mathrm{U}$ of Bst DNA polymerase large fragment (New England Biolabs, Massachusetts, USA), $2.5 \mu \mathrm{L} 10 \times$ ThermoPol Buffer, $4 \mathrm{mM} \mathrm{MgSO}_{4}, 1 \mathrm{mM}$ dNTPs (Aidlab, Beijing, China), $1.6 \mathrm{mM}$ each of FIP and BIP, $0.2 \mathrm{mM}$ each of F3 and B3, 0.94 M betaine (Aladdin, Shanghai, China), and $1 \mu \mathrm{L}$ of target DNA. Sterilized water was used as negative control. The mixture was incubated at $63^{\circ} \mathrm{C}$ for $60 \mathrm{~min}$, followed by heating at $80^{\circ} \mathrm{C}$ for $10 \mathrm{~min}$ to inactivate the enzyme and terminate the reaction. The LAMP products were analyzed by $1 \%$ agarose gel electrophoresis stained with GelRed (US Everbright Inc., Suzhou, China). Additionally, $0.2 \mu \mathrm{L}$ $10,000 \times$ SYBR Green I (Aidlab) was added (final concentration of SYBR Green I was $80 \times$ ) into the product to facilitate the visualization of the reaction by naked eyes. The experiment was conducted twice.

\section{Optimization of LAMP reaction}

The primer set Tub-E198V and relevant genomic DNA were chosen to optimize the LAMP reaction. The 
optimization was conducted in a total volume of $25 \mu \mathrm{L}$. A series of concentrations of $\mathrm{Mg}^{2+}(2,3,4,5$ and $6 \mathrm{mM})$, $\operatorname{dNTP}(0.5,1,1.5$ and $2 \mathrm{mM})$, betaine $(0,0.8$ and $1.6 \mathrm{M})$, Bst DNA polymerase (2, 4, 6 and $8 \mathrm{U})$, inner primers $(0.8,1.2,1.6$ and $2.0 \mu \mathrm{M})$ and outer primers $(0.2,0.4,0.6$ and $0.8 \mu \mathrm{M})$ were evaluated. First, the concentration of $\mathrm{Mg}^{2+}$ was optimized while all the other components remained constant. Then, the concentration of dNTPs was optimized at the optimal concentration of $\mathrm{Mg}^{2+}$. Accordingly, the concentrations of betaine, Bst DNA polymerase, inner primers and outer primers were determined, respectively. Moreover, using the optimized reaction components, the reaction was performed at $61^{\circ} \mathrm{C}, 62^{\circ} \mathrm{C}, 63^{\circ} \mathrm{C}$, $64{ }^{\circ} \mathrm{C}$ and $65^{\circ} \mathrm{C}$ for $60 \mathrm{~min}$, respectively, to examine the best temperature. Later, the reaction was carried out at the optimal temperature for 30, 45, 60, 75 and 90 min, respectively, to test the optimal time. The LAMP products were assessed based on agarose gel electrophoresis and color visualization with SYBR Green I as described above. The experiment was performed twice.

\section{Sensitivity of LAMP and conventional PCR}

The sensitivity of LAMP and PCR was analyzed using Tub-E198V primer set and ten-fold serial dilutions of plasmid pMD18-TubA, which contained the E198V fragment of TUB2. For construction of pMD18-TubA, the E198V fragment was obtained by PCR with primer pair TubA 1378F19/2314R20 (5'-GAT CTC CAA CTT GAG CGT A / TGG AAA CCT TAC CAC GGC TA-3'). The PCR was performed as described in the following sensitivity test and the product was purified using EasyPure PCR Purification Kit (TransGen, Beijing, China). The purified fragment was then cloned into pMD18-T vector using pMD18-T Vector Cloning kit (TaKaRa, Dalian, China). The recombinant plasmid pMD18-TubA was extracted using EasyPure Plasmid MiniPrep Kit (TransGen) and ten-fold serially diluted from $2 \times 10^{8}$ to $2 \times 10^{0}$ copies/ $\mu \mathrm{L}$ prior to use.

For conventional PCR detection, primer pair TubA 1378F19/2314R20 was used to amplify a 936-bp DNA fragment. PCR amplication was performed in a total reaction volume of $25 \mu \mathrm{L}$, containing $1.25 \mathrm{U}$ of EasyTaq DNA Polymerase (TransGen), $1 \times$ EasyTaq buffer, 1.25 $\mathrm{mM}$ each dNTP, $0.4 \mu \mathrm{M}$ each primer, and $1 \mu \mathrm{L}$ corresponding amount of template DNA. Amplification was carried out in an iCycler Thermal Cycler (Bio-Rad Laboratories Inc., Hercules, CA). The PCR program consisted of an initial preheating step at $94^{\circ} \mathrm{C}$ for $3 \mathrm{~min}$, followed by 35 cycles of denaturation at $94^{\circ} \mathrm{C}$ for $30 \mathrm{~s}$, annealing at $58^{\circ} \mathrm{C}$ for $30 \mathrm{~s}$, and extension at $72^{\circ} \mathrm{C}$ for 60 s, with a final extension step at $72^{\circ} \mathrm{C}$ for $10 \mathrm{~min}$. For LAMP detection, LAMP reaction was performed at reaction conditions optimized as described. The PCR product was separated on $1 \%$ agarose gel stained with ethidium bromide $(\mathrm{EB})$ in $1 \times$ TAE buffer and visualized under UV light. The LAMP products were examined by both agarose gel electrophoresis and the color change of SYBR Green I. The analysis was conducted twice.

\section{Specificity and reproducibility test of LAMP}

Primer set Tub-E198V was also tested against other relative species of $B$. cinerea, including $B$. sinoviticola, $B$. sinoallii, B. porri, S. sclerotiorum, M. fructicola, $M$. mumecola, M. yunnanensis, and F. graminearum, to determine the specificity of LAMP primers. Additionally, all three LAMP primer sets designed in this study were applied to evaluate the reproducibility using genomic DNA extracted from eight for each of wild type, E198V, E198A, and E198K isolates. Instead of DNA template, sterile water was used in negative control, while other reaction components remained identical. The LAMP was conducted at the optimal reaction parameters and the product was assessed as described above. All experiments were conducted twice.

\section{LAMP application using mycelia}

Four isolates for each of wild-type B. cinerea (sensitive to benzimidazole), and its benzimidazole-resistant phenotypes E198V, E198A, and E198K were analyzed in this test. Mycelia on approximately $0.5 \mathrm{~cm}^{2}$ agar medium were prepared using toothpicks after culturing on PDA plates for a week. Then, the mycelia were suspended in $50 \mu \mathrm{L} 10 \times$ Tris-EDTA (TE) buffer $(100 \mathrm{mM}$ Tris- $\mathrm{HCl}$, $10 \mathrm{mM}$ EDTA, pH 8.0) in a $1.5 \mathrm{~mL}$ centrifuge tube. After boiling in water for $2 \mathrm{~min}$, the suspension was chilled on ice for $2 \mathrm{~min}$ and then centrifuged at $12000 \mathrm{rpm}$ for 1 min. The supernatants obtained were used as templates for LAMP. The LAMP assay was conducted and assessed with the primer set Tub-E198V using the optimal LAMP reaction parameters as described above. The experiment was performed twice.

\section{Additional files}

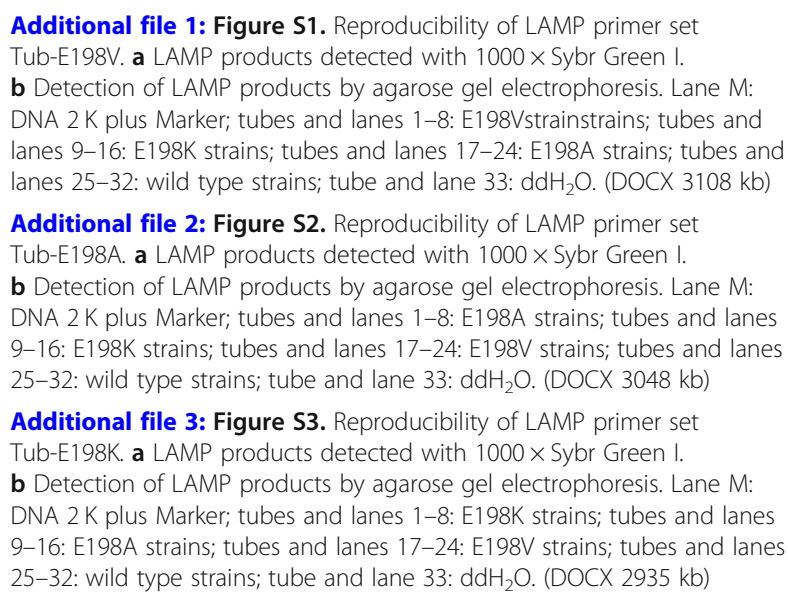

Additional file 2: Figure S2. Reproducibility of LAMP primer set Tub-E198A. a LAMP products detected with $1000 \times$ Sybr Green I. b Detection of LAMP products by agarose gel electrophoresis. Lane M: DNA 2 K plus Marker; tubes and lanes 1-8: E198A strains; tubes and lanes 9-16: E198K strains; tubes and lanes 17-24: E198V strains; tubes and lanes 25-32: wild type strains; tube and lane 33: $\mathrm{ddH}_{2} \mathrm{O}$. (DOCX $3048 \mathrm{~kb}$ )

Additional file 3: Figure S3. Reproducibility of LAMP primer set Tub-E198K. a LAMP products detected with $1000 \times$ Sybr Green I. b Detection of LAMP products by agarose gel electrophoresis. Lane M: DNA 2 K plus Marker; tubes and lanes 1-8: E198K strains; tubes and lanes 9-16: E198A strains; tubes and lanes 17-24: E198V strains; tubes and lanes 25-32: wild type strains; tube and lane 33: dd $\mathrm{H}_{2} \mathrm{O}$. (DOCX 2935 kb) 


\section{Abbreviations}

E198A, V, K: Substitutions from glutamic acid to alanine, valine, lysine at codon 198 of TUB2; EB: Ethidium bromide; F200Y: Mutation at codon 200 of TUB2 substituting phenylalanine by tyrosine; HNB: Hydroxynaphthol blue; LAMP: Loop-mediated isothermal amplification; MBCs: Benzimidazole fungicides; PCR-RFLP: PCR-restriction-fragment length polymorphism; PDA: Potato dextrose agar; TUB2: ß-tubulin gene

\section{Acknowledgements}

We are grateful to Miss $\mathrm{Na} \mathrm{Li}$, a former graduate student of Professor Guo-Qing Li for providing us the isolates used in this study.

\section{Funding}

This work is supported by the Special Fund for Agro-scientific Research in the Public Interest (Nos. 201303025 and 201303023).

\section{Availability of data and materials}

The datasets used and/or analyzed during the current study are available from the corresponding author on reasonable request.

\section{Authors' contributions}

FF and C-XL designed the research; FF performed research; FF, MH, G-QL, YL and $\mathrm{C}-\mathrm{XL}$ wrote the manuscript, all authors read and approved the final manuscript.

\section{Ethics approval and consent to participate}

Not applicable.

\section{Consent for publication}

Not applicable.

\section{Competing interests}

The authors declare that they have no competing interests.

\section{Author details}

${ }^{1}$ Key Lab of Horticultural Plant Biology, Ministry of Education, and College of Plant Science and Technology, Huazhong Agricultural University, Wuhan 430070, China. ${ }^{2}$ Department of Biology, University of Kaiserslautern, 67653 Kaiserslautern, Germany. ${ }^{3}$ College of Plant Science and Technology and the Key Lab of Crop Disease Monitoring \& Safety Control in Hubei Province, Huazhong Agricultural University, Wuhan 430070, China.

Received: 8 October 2018 Accepted: 12 February 2019

Published online: 04 March 2019

\section{References}

Angamuthu R, Baskaran S, Gopal DR, Devarajan J, Kathaperumal K. Rapid detection of the Marek's disease viral genome in chicken feathers by loopmediated isothermal amplification. J Clin Microbiol. 2012;50:961-5.

Banno S, Fukumori F, Ichiishi A, Okada K, Uekusa H, Kimura M, et al. Genotyping of benzimidazole-resistant and dicarboximide-resistant mutations in Botrytis cinerea using real-time polymerase chain reaction assays. Phytopathology. 2008:98:397-404.

Bardas GA, Myresiotis CK, Karaoglanidis GS. Stability and fitness of anilinopyrimidine-resistant strains of Botrytis cinerea. Phytopathology. 2008;98: 443-50.

Bolton AT. Fungicide resistance in Botrytis cinerea, the result of selective pressure on resistant strains already present in nature. Can J Plant Sci. 1976;56:861-4.

Chatzidimopoulos M, Ganopoulos I, Madesis P, Vellios E, Tsaftaris A, Pappas AC. High-resolution melting analysis for rapid detection and characterization of Botrytis cinerea phenotypes resistant to fenhexamid and boscalid. Plant Pathol. 2014;63:1336-43.

Chi MH, Park SY, Lee YH. A quick and safe method for fungal DNA extraction. Plant Pathol J. 2009:25:108-11.

Côté M-J, Tardif M-C, Meldrum AJ. Identification of Monilinia fructigena, M. fructicola, M. laxa, and Monilia polystroma on inoculated and naturally infected fruit using multiplex PCR. Plant Dis. 2004;88:1219-25.

Duan $Y$, Yang $Y$, Wang $Y$, Pan $X$, Wu J, Cai Y, et al. Loop-mediated isothermal amplification for the rapid detection of the F200Y mutant genotype of carbendazim-resistant isolates of Sclerotinia sclerotiorum. Plant Dis. 2016;100: 976-83.
Duan Y, Zhang X, Ge C, Wang Y, Cao J, Jia X, et al. Development and application of loop-mediated isothermal amplification for detection of the F167Y mutation of carbendazim-resistant isolates in Fusarium graminearum. Sci Rep. 2014a;4:7094.

Duan YB, Ge CY, Zhang XK, Wang JX, Zhou MG. Development and evaluation of a novel and rapid detection assay for Botrytis cinerea based on loopmediated isothermal amplification. PLoS One. 2014b;9:e111094.

Duan YB, Yang Y, Wang JX, Liu CC, He LL, Zhou MG. Development and application of loop-mediated isothermal amplification for detecting the highly benzimidazole-resistant isolates in Sclerotinia sclerotiorum. Sci Rep. 2015;5:17278

Fan F, Hamada MS, Li N, Li G, Luo C. Multiple fungicide resistance in Botrytis cinerea from greenhouse strawberries in Hubei Province, China. Plant Dis. 2017;101:601-6.

Fan F, Li N, Li GQ, Luo CX. Occurrence of fungicide resistance in Botrytis cinerea from greenhouse tomato in Hubei Province, China. Plant Dis. 2016;100:2414-21.

Fernández-Ortuño D, Grabke A, Bryson PK, Amiri A, Peres NA, Schnabel G. Fungicide resistance profiles in Botrytis cinerea from strawberry fields of seven southern U.S. states. Plant Dis. 2014;98:825-33.

Goto M, Honda E, Ogura A, Nomoto A, Hanaki K. Colorimetric detection of loopmediated isothermal amplification reaction by using hydroxy naphthol blue. BioTechniques. 2009;46:167-72.

Hahn M. The rising threat of fungicide resistance in plant pathogenic fungi: Botrytis as a case study. J Chem Biol. 2014;7:133-41.

Hu M-J, Cox KD, Schnabel G, Luo C-X. Monilinia species causing brown rot of peach in China. PLoS One. 2011;6:e24990.

Karthik K, Rathore R, Thomas P, Arun TR, Viswas KN, Dhama K, et al. New closed tube loop mediated isothermal amplification assay for prevention of product cross-contamination. MethodsX. 2014;1:137-43.

Kuan C-P, Lin Y-F, Lin Y-C, Chen C-C. One-step reverse transcription loopmediated isothermal amplification for the detection of Capsicum chlorosis virus in Phalaenopsis orchids. J Gen Plant Pathol. 2016:82:307-13.

Leroux P, Chapeland F, Desbrosses D, Gredt M. Patterns of cross-resistance to fungicides in Botryotinia fuckeliana (Botrytis cinerea) isolates from French vineyards. Crop Protect. 1999;18:687-97.

Li F, Yan W, Long L, Qi X, Li C, Zhang S. Development and application of loopmediated isothermal amplification assays for rapid visual detection of cry2Ab and cry3A genes in genetically-modified crops. Int J Mol Sci. 2014;15:15109-21.

Luck JE, Gillings MR. Rapid identification of benomyl resistant strains of Botrytis cinerea using the polymerase chain reaction. Mycol Res. 1995;99:1483-8.

Moradi A, Nasiri J, Abdollahi H, Almasi M. Development and evaluation of a loopmediated isothermal amplification assay for detection of Erwinia amylovora based on chromosomal DNA. Eur J Plant Pathol. 2012:133:609-20.

Mori Y, Kanda H, Notomi T. Loop-mediated isothermal amplification (LAMP): recent progress in research and development. J Infect Chemother. 2013;19: 404-11.

Nagamine K, Hase T, Notomi T. Accelerated reaction by loop-mediated isothermal amplification using loop primers. Mol Cell Probes. 2002;16:223-9.

Niessen L, Vogel RF. Detection of Fusarium graminearum DNA using a loopmediated isothermal amplification (LAMP) assay. Int J Food Microbiol. 2010; 140:183-91.

Njiru ZK. Loop-mediated isothermal amplification technology: towards point of care diagnostics. PLoS Negl Trop Dis. 2012;6:e1572.

Njiru ZK, Mikosza AS, Armstrong T, Enyaru JC, Ndung'u JM, Thompson AR. Loopmediated isothermal amplification (LAMP) method for rapid detection of Trypanosoma brucei rhodesiense. PLoS Negl Trop Dis. 2008;2:e147.

Notomi T, Okayama H, Masubuchi H, Yonekawa T, Watanabe K, Amino N, et al. Loop-mediated isothermal amplification of DNA. Nucleic Acids Res. 2000;28:e63.

Pan L, Li J, Zhang WN, Dong L. Detection of the 11781L mutation in fenoxapropp-ethyl-resistant American sloughgrass (Beckmannia syzigachne Steud.), based on the loop-mediated isothermal amplification method. Pest Manag Sci. 2015;71:123-30.

Tomita N, Mori Y, Kanda H, Notomi T. Loop-mediated isothermal amplification (LAMP) of gene sequences and simple visual detection of products. Nat Protoc. 2008;3:877-82.

Tomlinson JA, Dickinson MJ, Boonham N. Detection of Botrytis cinerea by loopmediated isothermal amplification. Lett Appl Microbiol. 2010a;51:650-7.

Tomlinson JA, Dickinson MJ, Boonham N. Rapid detection of Phytophthora ramorum and $P$. kernoviae by two-minute DNA extraction followed by isothermal amplification and amplicon detection by generic lateral flow device. Phytopathology. 2010b;100:143-9. 
Tripathi RK, Schlösser E. The mechanism of resistance of Botrytis cinerea to methyl benzimidazol-2-yl carbamate (MBC). J Plant Dis Protect. 1982;89:151-6.

Walker A-S, Micoud A, Rémuson F, Grosman J, Gredt M, Leroux P. French vineyards provide information that opens ways for effective resistance management of Botrytis cinerea (grey mould). Pest Manag Sci. 2013;69:667-78.

Wang D, Zhang G, Lu C, Deng R, Zhi A, Guo J, et al. Rapid detection of Listeria monocytogenes in raw milk with loop-mediated isothermal amplification and chemosensor. J Food Sci. 2011;76:M611-5.

Wang F, Jiang L, Ge B. Loop-mediated isothermal amplification assays for detecting Shiga toxin-producing Escherichia coli in ground beef and human stools. J Clin Microbiol. 2012;50:91-7.

Weber RWS. Resistance of Botrytis cinerea to multiple fungicides in northern German small-fruit production. Plant Dis. 2011;95:1263-9.

Williamson B, Tudzynski B, Tudzynski P, van Kan JA. Botrytis cinerea: the cause of grey mould disease. Mol Plant Pathol. 2007:8:561-80.

Yarden O, Katan T. Mutations leading to substitutions at amino acids 198 and 200 of beta-tubulin that correlate with benomyl-resistance phenotypes of field strains of Botrytis cinerea. Phytopathology. 1993;83:1478-83.

Zhang CQ, Liu YH, Zhu GN. Detection and characterization of benzimidazole resistance of Botrytis cinerea in greenhouse vegetables. Eur J Plant Pathol. 2010;126:509-15.

Zhou D, Guo J, Xu L, Gao S, Lin Q, Wu Q, et al. Establishment and application of a loop-mediated isothermal amplification (LAMP) system for detection of cry1Ac transgenic sugarcane. Sci Rep. 2014;4:4912.

Ziogas BN, Nikou D, Markoglou AN, Malandrakis AA, Vontas J. Identification of a novel point mutation in the $\beta$-tubulin gene of Botrytis cinerea and detection of benzimidazole resistance by a diagnostic PCR-RFLP assay. Eur J Plant Pathol. 2009;125:97-107.

Zoheir KM, Allam AA. A rapid improved method for sexing embryo of water buffalo. Theriogenology. 2011;76:83-7.

Ready to submit your research? Choose BMC and benefit from:

- fast, convenient online submission

- thorough peer review by experienced researchers in your field

- rapid publication on acceptance

- support for research data, including large and complex data types

- gold Open Access which fosters wider collaboration and increased citations

- maximum visibility for your research: over $100 \mathrm{M}$ website views per year

At $\mathrm{BMC}$, research is always in progress.

Learn more biomedcentral.com/submissions 